\title{
The maximal quartile operator
}

Christoph Thiele

\section{Introduction.}

Let $C^{\Delta}(\mathbb{R})$ denote the set of all functions $f: \mathbb{R} \longrightarrow \mathbb{R}$ that are finite linear combinations of characteristic functions of dyadic intervals, i.e., intervals of the form $\left[2^{k} n, 2^{k}(n+1)\right)$ with $k, n \in \mathbb{Z}$. We define the Walsh function $W_{l} \in C^{\Delta}(\mathbb{R})$ for $l \in \mathbb{N}_{0}$ by the following recursive formulas

$$
\begin{gathered}
W_{0}=\mathbf{1}_{[0,1)}, \\
W_{2 l}=W_{l}(2 x)+W_{l}(2 x-1), \\
W_{2 l+1}=W_{l}(2 x)-W_{l}(2 x-1) .
\end{gathered}
$$

For $k, n \in \mathbb{Z}, l \in \mathbb{N}_{0}$ we define the Walsh wave packet $w_{k, n, l}$ by

$$
w_{k, n, l}(x)=2^{-k / 2} W_{l}\left(2^{-k} x-n\right) .
$$

The quartile operator $H_{W}$ and the maximal quartile operator $H_{W}^{\max }$ are then defined by

$$
\begin{gathered}
H_{W}(f, g):=\sum_{\substack{k, n \in \mathbb{Z} \\
l \in \mathbb{N}_{0}}} 2^{-k / 2}\left\langle f, w_{k, n, 4 l}\right\rangle\left\langle g, w_{k, n, 4 l+1}\right\rangle w_{k, n, 4 l+2}, \\
H_{W}^{\max }(f, g)(x) \\
:=\sup _{K \in \mathbb{Z}}\left|\sum_{\substack{k, n \in \mathbb{Z} \\
l \in \mathbb{N}_{0} \\
k \leq K}} 2^{-k / 2}\left\langle f, w_{k, n, 4 l}\right\rangle\left\langle g, w_{k, n, 4 l+1}\right\rangle w_{k, n, 4 l+2}(x)\right| .
\end{gathered}
$$


In this paper we prove the following theorem:

Theorem 1. Let $p, q, r$ satisfy

$$
\frac{1}{p}=\frac{1}{q}+\frac{1}{r}, \quad \frac{2}{3}<p<\infty, 1<q, r \leq \infty .
$$

Then there is a constant $C$ such that for all functions $f, g \in C^{\Delta}(\mathbb{R})$

$$
\begin{gathered}
\left\|H_{W}(f, g)\right\|_{p} \leq C\|f\|_{q}\|g\|_{r} \\
\left\|H_{W}^{\max }(f, g)\right\|_{p} \leq C\|f\|_{q}\|g\|_{r}
\end{gathered}
$$

Only the estimates for $H_{W}^{\max }$ are new, but our approach gives the estimates for $H_{W}$ without extra work.

The quartile operator has been introduced in [11] as a discrete model for the bilinear Hilbert transform. The bilinear Hilbert transform $H$ is defined as a bilinear operation from $\mathcal{S}(\mathbb{R}) \times \mathcal{S}(\mathbb{R})$ into $C(\mathbb{R})$ by

$$
H(f, g)(x):=\text { p.v. } \int f(x-t) g(x+t) \frac{d t}{t} .
$$

It has been shown in [5] and [7], see also [6] and [8] for a survey and [11] for a condensed proof, that the bilinear Hilbert transform satisfies the a priori estimates

$$
\|H(f, g)\|_{p} \leq C_{q, r}\|f\|_{q}\|g\|_{r}
$$

provided $p, q, r$ satisfy (4). More recently, M. Lacey has shown (see [4]) that also the maximal truncation of the bilinear Hilbert transform,

$$
H^{\max }(f, g)(x):=\sup _{\varepsilon>0}\left|\int_{\mathbb{R} \backslash[-\varepsilon, \varepsilon]} f(x-t) g(x+t) \frac{d t}{t}\right|,
$$

satisfies estimates as in (5), (4). By the same method he has observed that the maximal operator

$$
M(f, g)(x):=\sup _{\varepsilon>0}\left|\frac{1}{\varepsilon} \int_{[-\varepsilon, \varepsilon]} f(x-t) g(x+t) d t\right|
$$

satisfies estimates as in (5), (4). For the operator $M$, these estimates are nontrivial only if $p \leq 1$. 
The current paper is an adaption of the ideas in [4] to the discrete model of the quartile operator. As in [4], the main ingredient that is needed to pass from estimates for $H_{W}$ to estimates for $H_{W}^{\max }$ is a version of a lemma by Bourgain (see [1]) for certain maximal averages.

We use analysis in the Walsh phase plane as in [11]. We give all the necessary definitions, but at some places we refer to results in [11].

\section{The main lemma.}

The main issue in proving Theorem 1 is to efficiently make use of orthogonality of wave packets. For this we have to identify appropriate large sets of pairwise orthogonal wave packets. We will associate to each wave packet a rectangle in the half plane, so that disjoint rectangles correspond to orthogonal wave packets. Then the combinatorial issue is to identify sets of pairwise disjoint rectangles. This is the main idea behind the following Lemma 1. In the proof of this lemma one has to identify sets of pairwise disjoint rectangles so that we can use the second hypothesis of the lemma. This lemma already appears implicitly in [7].

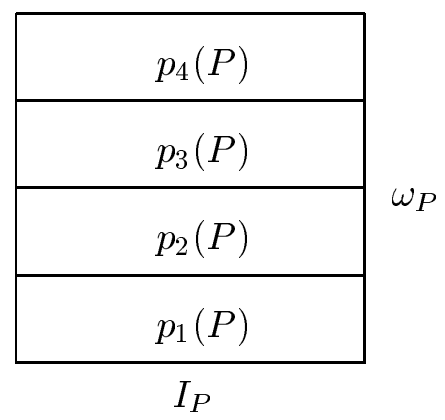

Table 1. Subdivision of quartiles.

A tile $p$ is a rectangle $p=I_{p} \times \omega_{p}$ of area one in the upper half plane, such that $I_{p}$ and $\omega_{p}$ are dyadic. Hence for each tile $p$ there are integers $k, n, l$ with $l \geq 0$ such that

$$
I_{p}=\left[2^{k} n, 2^{k}(n+1)\right), \quad \omega_{p}=\left[2^{-k} l, 2^{-k}(l+1)\right) .
$$

Similarly, a quartile $P$ is a rectangle $I_{P} \times \omega_{P}$ of area four in the upper half plane, such that $I_{P}$ and $\omega_{P}$ are dyadic. Each quartile $P$ is the union of four tiles $p_{1}(P), p_{2}(P), p_{3}(P)$, and $p_{4}(P)$, as in Figure 1 . 
If $p, q$ are two tiles, then we write $p<q$ if $I_{p} \subset I_{q}$ and $\omega_{q} \subset \omega_{p}$. This defines a partial ordering of the set of tiles. Let $\imath \in\{1,2,3\}$. A set $T$ of quartiles is called a tree of type $\imath$, if $\left\{p_{\imath}(P)\right\}_{P \in T}$ contains exactly one element which is maximal in $\left\{p_{\imath}(P)\right\}_{P \in T}$. If $p_{\imath}\left(P_{T}\right)$ is this maximal element, we write $p_{\imath}\left(P_{T}\right)=I_{T} \times \omega_{T}=p_{T}$ and call $p_{T}$ the top of the tree.

Lemma 1. Assume that we are given exponents $1 \leq s_{\jmath}<\infty$ for $\jmath \in\{1,2,3\}$ such that $1 / s_{1}+1 / s_{2}+1 / s_{3}>1$, and we are given $a$ constant $B>0$. Then there is a constant $C>0$ such that the following holds:

Let $\mathbf{P}$ be a finite set of quartiles. For each $\jmath \in\{1,2,3\}$ let

$$
a_{\jmath}: \mathbf{P} \longrightarrow \mathbb{R}^{+}
$$

be a function such that the following two hypotheses are satisfied:

1) Let $\imath \neq \jmath$. If $T \subset \mathbf{P}$ is a tree of type $\imath$, then

$$
\left\|\left(\sum_{P \in T} \frac{a_{\jmath}(P)^{2}}{\left|I_{P}\right|} \mathbf{1}_{I_{P}}\right)^{1 / 2}\right\|_{1} \leq B\left|I_{T}\right| .
$$

2) Let $\imath \neq \jmath, m \in \mathbb{Z}$. Let $\mathbf{P}^{\prime} \subset \mathbf{P}$ be a disjoint union of trees of type $\imath$

$$
\mathbf{P}^{\prime}:=\bigcup_{T \in \mathcal{F}} T
$$

such that the set $\left\{p_{\jmath}(P): P \in \mathbf{P}^{\prime}\right\}$ is a set of pairwise disjoint rectangles and, for each $T \in \mathcal{F}$, we have

$$
\left\|\left(\sum_{P \in T} \frac{a_{\jmath}(P)^{2}}{\left|I_{P}\right|} \mathbf{1}_{I_{P}}\right)^{1 / 2}\right\|_{1} \geq 2^{m / s_{\jmath}}\left|I_{T}\right| .
$$

Then

$$
\sum_{T \in \mathcal{F}}\left|I_{T}\right| \leq B 2^{-m}
$$

Then we have the estimate

$$
\sum_{P \in \mathbf{P}}\left|I_{P}\right|^{-1 / 2} a_{1}(P) a_{2}(P) a_{3}(P) \leq C
$$


We prove the lemma. Let $m \in \mathbb{Z}$ and $\imath, \jmath \in\{1,2,3\}$ with $\imath \neq \jmath$. We say that a tree $T$ satisfies the size condition $(m, \imath, \jmath)$, if $T$ is of type $\imath$ and

$$
\left\|\left(\sum_{P \in T} \frac{a_{\jmath}(P)^{2}}{\left|I_{P}\right|} \mathbf{1}_{I_{P}}\right)^{1 / 2}\right\|_{1} \geq 2^{m / s_{\jmath}+3}\left|I_{T}\right| .
$$

We say that a tree $T$ satisfies the size condition $(m, \imath, \imath)$, if $T$ is of type $\imath$ and

$$
\frac{a_{\imath}(P)}{\sqrt{\left|I_{P}\right|}} \geq 2^{m / s_{\imath}}
$$

for all $P \in T$.

The size of a tree $T$ is the maximal $m \in \mathbb{Z}$ such that $T$ satisfies a size condition $(m, \imath, \jmath)$ for some $\imath, \jmath \in\{1,2,3\}$.

We partition the set $\mathbf{P}$ into trees $T_{0}, \ldots, T_{N}$ as follows. Let $\nu \in \mathbb{N}_{0}$ and assume by induction that $T_{\nu^{\prime}}$ is already chosen for all $\nu^{\prime}$ with $\nu^{\prime} \leq \nu$. Define

$$
\mathbf{P}_{\nu}:=\mathbf{P} \backslash \bigcup_{\nu^{\prime} \leq \nu} T_{\nu^{\prime}}
$$

We can assume $\mathbf{P}_{\nu}$ is not empty. Let $m_{\nu}$ be the maximal integer for which there exists a tree $T \subset \mathbf{P}_{\nu}$ of size $m_{\nu}$, and let $\mathcal{F}_{\nu}$ be the set of all trees $T \subset \mathbf{P}_{\nu}$ of size $m_{\nu}$. Define $\mathcal{F}_{\nu}^{\text {max }}$ to be the set of trees in $\mathcal{F}_{\nu}$ which are maximal in $\mathcal{F}_{\nu}$ with respect to set inclusion. Let $\mathcal{F}_{\nu,<}$ be the set of all trees in $\mathcal{F}_{\nu}^{\max }$ which satisfy a size condition $(m, \imath, \jmath)$ with

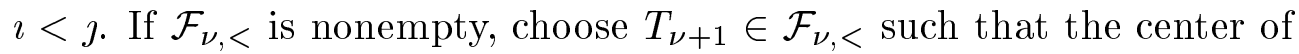
$\omega_{T_{\nu+1}}$ is maximal. If $\mathcal{F}_{\nu,<}$ is empty, choose $T_{\nu+1} \in \mathcal{F}_{\nu}^{\max }$ such that the center of $\omega_{T_{\nu+1}}$ is minimal.

Since $\mathbf{P}$ is finite, the algorithm stops with a finite partition of $\mathbf{P}$ into $\left\{T_{1}, \ldots, T_{N}\right\}$. Define $\mathcal{F}:=\left\{T_{1}, \ldots, T_{N}\right\}$.

In the following estimates, $C$ will denote a constant depending on $s_{\jmath}$ and $B$. The precise value of $C$ may change from line to line.

Lemma 2. If $T \in \mathcal{F}$ and the size of $T$ is $m$, then, for $\imath \neq \jmath$,

$$
\left(\sum_{P \in T} a_{\jmath}(P)^{2}\right)^{1 / 2} \leq C 2^{m / s_{\jmath}}\left|I_{T}\right|^{1 / 2},
$$

and

$$
\sup _{P \in T} \frac{a_{\imath}(P)}{\sqrt{\left|I_{P}\right|}} \leq C 2^{m / s_{\imath}}
$$


Proof. Let $T \in \mathcal{F}$ be of size $m$. Define

$$
f:=\left(\sum_{P \in T} \frac{a_{\jmath}(P)^{2}}{\left|I_{P}\right|} 1_{I_{P}}\right)^{1 / 2} .
$$

To prove the first estimate of the lemma, we have to bound the $L^{2}$ norm of $f$. We prove $\|f\|_{\mathrm{BMO}^{\Delta}} \leq C 2^{m / s_{3}}$, which gives the appropriate bound on $\|f\|_{2}$, because $f$ is supported on $I_{T}$.

Let $J$ be a dyadic interval. We have to show

$$
\inf _{c} \frac{1}{|J|} \int_{J}(f(x)-c) d x \leq C 2^{m / s_{\jmath}} .
$$

We split the sum in the definition of $f$ into the sum over those $P$ with $I_{P} \subset J$ and the sum over those $P$ with $I_{P} \not \subset J$. The second sum is constant on the interval $J$. Hence, using the inequality

$$
(a+b)^{1 / 2}-b^{1 / 2} \leq a^{1 / 2},
$$

which holds for any two positive numbers $a, b$, we can estimate the left hand side of inequality (8) by

$$
\frac{1}{|J|} \int_{J}\left(\sum_{P \in T: I_{P} \subset J} \frac{a_{\jmath}^{2}(P)}{\left|I_{P}\right|} \mathbf{1}_{I_{P}}(x)\right)^{1 / 2} d x .
$$

By passing to subintervals, if necessary, one observes that it suffices to bound this expression under the assumption that there is a $P^{\prime} \in T$ such that $I_{P^{\prime}}=J$. But then the set $T_{J}:=\left\{P \in T: I_{P} \subset J\right\}$ is a tree of type $\imath$. The size of this tree is at most $m$ by construction of the tree $T$. The size estimate for $T_{J}$ then shows that (9) is bounded by $C 2^{m / s_{j}}$. This finishes the desired BMO estimate and therefore the proof of the first estimate of the lemma. The second estimate follows immediately from the observation that the set $\{P\}$ is a tree of type $\imath$ for all $P \in T$ and has size less than or equal $m$. This finishes the proof of Lemma 2 .

Lemma 3. Let $\mathcal{F}_{m}$ be the set of trees in $\mathcal{F}$ with size $m$. Then

$$
\sum_{T \in \mathcal{F}^{m}}\left|I_{T}\right| \leq C 2^{-m}
$$


Proof. Fix $\imath, \jmath \in\{1,2,3\}$. It suffices to show the desired estimate for the sum over the set $\mathcal{F}_{m, \imath, \jmath}$ of those $T \in \mathcal{F}_{n}$ which satisfy the size condition $(m, \imath, \jmath)$ but no size condition $\left(m, \imath, \jmath^{\prime}\right)$ with $\jmath<\jmath^{\prime}$. We first consider the case $\imath=\jmath$. Pick $\imath^{\prime} \neq \imath$ and consider the set $\mathbf{p}_{m, \imath, \jmath}$ of all tiles which are tops of trees in $\mathcal{F}_{m, \imath, \jmath}$. Then $\mathbf{p}_{m, \imath, \jmath}$ is a set of pairwise disjoint rectangles. To see this assume to the contrary that the tops of two trees $T, T^{\prime} \in \mathcal{F}_{m, \imath, j}$ intersect. We can assume that $T$ has been selected before $T^{\prime}$. Then the union $T \cup T^{\prime}$ is a tree containing $T$, which contradicts the maximality of $T$ at the time it was selected. Since each set $\{P\}$ with $P \in \mathbf{P}^{\prime}$ is both a tree of type $\imath^{\prime}$ and of type $\imath$, we can apply (7) and the second hypothesis of the proposition to conclude the desired estimate.

Now assume $\imath<\jmath$. For a tree $T$ define $T^{\text {red }}$ to be the set of $P \in T$ such that $I_{P}$ is not minimal in $\left\{I_{P^{\prime}}: P^{\prime} \in T\right\}$. If $T^{\text {red }}$ is nonempty, it is again a tree. Define $T^{\text {Red }}=\left(T^{\text {red }}\right)^{\text {red }}$. If $T \in \mathcal{F}_{m, \imath, j}$, then

$$
\begin{aligned}
& \left\|\left(\sum_{P \in T^{\mathrm{Red}}} \frac{a_{\jmath}(P)^{2}}{\left|I_{P}\right|} \mathbf{1}_{I_{P}}\right)^{1 / 2}\right\|_{1} \\
& \quad \geq\left\|\left(\sum_{P \in T} \frac{a_{\jmath}(P)^{2}}{\left|I_{P}\right|} \mathbf{1}_{I_{P}}\right)^{1 / 2}\right\|_{1}-\left\|\left(\sum_{P \in T \backslash T^{\mathrm{Red}}} \frac{a_{\jmath}(P)^{2}}{\left|I_{P}\right|} \mathbf{1}_{I_{P}}\right)^{1 / 2}\right\|_{1} .
\end{aligned}
$$

Since the size of each tree $\{P\}$ with $P \in T \backslash T^{\text {Red }}$ is less than or equal $m$ and the intervals $I_{P}$ with $P \in T \backslash T^{\text {red }}$ as well as those with $P \in T^{\mathrm{red}} \backslash T^{\mathrm{Red}}$ are pairwise disjoint, we can bound this expression by

$$
\geq 2^{m / s_{\jmath}+3}\left|I_{T}\right|-2^{m / s_{\jmath}+1}\left|I_{T}\right|-2^{m / s_{\jmath}+1}\left|I_{T}\right| \geq 2^{m / s_{\jmath}}\left|I_{T}\right| .
$$

The desired estimate now follows from the second hypothesis of the proposition as soon as we prove that for any $T, T^{\prime} \in \mathcal{F}_{m, \imath, \jmath}$ and any $P \in T^{\mathrm{red}}, P^{\prime} \in T^{\prime \text { red }}$ with $P \neq P^{\prime}$ we have that $p_{\jmath}(P)$ and $p_{\jmath}\left(P^{\prime}\right)$ are

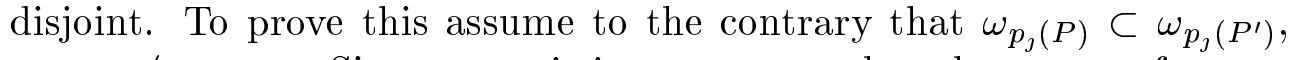
$\omega_{p_{\jmath}(P)} \neq \omega_{p_{\jmath}\left(P^{\prime}\right)}$. Since $\imath<\jmath$, it is easy to see that the center of $\omega_{p_{\imath}(P)}$ is greater than the center of $\omega_{p_{\imath}\left(P^{\prime}\right)}$. Hence $T$ has been selected before $T^{\prime}$. Pick $P^{\prime \prime}, P^{\prime \prime \prime} \in T^{\prime} \backslash T^{\prime \text { Red }}$ such that $p_{\imath}\left(P^{\prime \prime}\right)<p_{\imath}\left(P^{\prime \prime \prime}\right)<p_{\imath}\left(P^{\prime}\right)$. Then we have

$$
\omega_{p_{\imath}(P)} \subset \omega_{p_{\imath}\left(P^{\prime \prime}\right)}, \quad I_{p_{\imath}\left(P^{\prime \prime}\right)} \subset I_{p_{\imath}(P)} .
$$

Hence $P^{\prime \prime}$ qualifies to be in the tree $T$, a contradiction to the maximality of $T$. This finishes the proof of Lemma 3 , since the case $\imath>\jmath$ is done similarly to the case $\imath<\jmath$. 
The size of a tree in $\mathcal{F}$ is bounded by a constant $C$. This is immediate in the case of size conditions $(m, \imath, \jmath)$ with $\imath \neq \jmath$ from the first hypothesis of the lemma. For $\imath=\jmath$ we apply, as we have done before, the first hypothesis of the lemma to trees containing just one element.

Hence we have

$$
\begin{aligned}
& \sum_{P \in \mathbf{P}} \frac{1}{\sqrt{\left|I_{P}\right|}} a_{1}(P) a_{2}(P) a_{3}(P) \\
&=\sum_{\substack{m \in \mathbb{Z} \\
m \leq C}} \sum_{\substack{m, \jmath=1 \\
m \in \mathcal{F}_{m, \imath, \jmath}}}^{3} \sum_{P \in T} \frac{1}{\sqrt{\left|I_{P}\right|}} a_{1}(P) a_{2}(P) a_{3}(P) .
\end{aligned}
$$

Applying Hölder's inequality gives

$$
\cdots \leq \sum_{m \leq m_{0}} \sum_{\imath, \jmath=1}^{3} \sum_{T \in \mathcal{F}_{m, \imath, \jmath}} \sup _{P \in T} \frac{a_{\imath}(P)}{\sqrt{\left|I_{P}\right|}} \prod_{l \neq \imath}\left(\sum_{P \in T} a_{l}(P)^{2}\right)^{1 / 2} .
$$

Now Lemma 2 gives

$$
\cdots \leq \sum_{m \leq C} \sum_{\imath, J=1}^{3} \sum_{T \in \mathcal{F}_{(m, r, j)}} C 2^{\left(1 / s_{1}+1 / s_{2}+1 / s_{3}\right) m}\left|I_{T}\right| .
$$

Finally Lemma 3 gives

$$
\leq \sum_{m \leq C} \sum_{i, J=1}^{3} C 2^{\left(1 / s_{1}+1 / s_{2}+1 / s_{3}-1\right) m}
$$

This is a convergent geometric series and hence bounded by a constant $C$. This finishes the proof of Lemma 1 .

\section{The maximal quartile operator.}

If $p$ is the tile $\left[2^{k} n, 2^{k}(n+1)\right) \times\left[2^{-k} l, 2^{-k}(l+1)\right)$, then we denote by $w_{p}$ the Walsh wave packet given by

$$
w_{p}(x):=w_{k, n, l}(x)=2^{-k / 2} W_{l}\left(2^{-k} x-n\right) .
$$


The significance of this identification is that if $p$ and $p^{\prime}$ are two disjoint tiles, then $w_{p}$ and $w_{p^{\prime}}$ are orthogonal. Moreover if a $p<q$ for two tiles $p$ and $q$, then then on the interval $I_{p}$ the functions $w_{p}$ and $w_{q}$ are multiples of each other. For a proof of these easy facts see [11].

Let $\mathbf{P}$ denote the set of all quartiles. Then the maximal quartile operator $H_{W}^{\max }$ can be written as

$$
\begin{aligned}
& H_{W}^{\max }(f, g)(x) \\
& \quad:=\sup _{k \in \mathbb{Z}}\left|\sum_{P \in \mathbf{P}:\left|I_{P}\right| \geq 2^{k}} \frac{1}{\sqrt{\left|I_{P}\right|}}\left\langle w_{p_{1}(P)}, f\right\rangle\left\langle w_{p_{2}(P)}, g\right\rangle w_{p_{3}(P)}(x)\right| .
\end{aligned}
$$

Now let $\kappa \in C^{\Delta}(\mathbb{R})$. Then the linearized maximal quartile operator $H_{W}^{\kappa}$ is defined by

$$
H_{W}^{\kappa}(f, g)(x):=\sum_{P \in \mathbf{P}:\left|I_{P}\right| \geq 2^{\kappa(x)}} \frac{1}{\sqrt{\left|I_{P}\right|}}\left\langle w_{p_{1}(P)}, f\right\rangle\left\langle w_{p_{2}(P)}, g\right\rangle w_{p_{3}(P)}(x) .
$$

By standard arguments, an $L^{p}$-bound on $H_{W}^{\kappa}$ that does not depend on the function $\kappa$ implies the corresponding bound for $H_{W}^{\max }$. We fix the function $\kappa$ and write

$$
H_{W}^{\kappa}(f, g)(x)=\sum_{P \in \mathbf{P}} \frac{1}{\sqrt{\left|I_{P}\right|}}\left\langle v_{1, P}, f\right\rangle\left\langle v_{2, P}, g\right\rangle v_{3, P}(x),
$$

where

$$
\begin{aligned}
v_{1, P} & :=w_{p_{1}(P)}, \quad v_{2, P}:=w_{p_{2}(P)}, \\
v_{3, P}(x) & := \begin{cases}w_{p_{3}(P)}(x), & \text { if }\left|I_{P}\right| \geq 2^{\kappa(x)}, \\
0, & \text { if }\left|I_{P}\right|<2^{\kappa(x)} .\end{cases}
\end{aligned}
$$

By integrating against a third function $f_{3}$, we obtain a trilinear form

$$
\begin{gathered}
T_{W}^{\kappa}: C^{\Delta}(\mathbb{R}) \times C^{\Delta}(\mathbb{R}) \times C^{\Delta}(\mathbb{R}) \longrightarrow \mathbb{R}, \\
T_{W}^{\kappa}\left(f_{1}, f_{2}, f_{3}\right)=\sum_{P \in \mathbf{P}} \frac{1}{\sqrt{\left|I_{P}\right|}}\left\langle v_{1, P}, f_{1}\right\rangle\left\langle v_{2, P}, f_{2}\right\rangle\left\langle v_{3, P}, f_{3}\right\rangle .
\end{gathered}
$$

For each permutation $\sigma$ of the set $\{1,2,3\}$ we obtain the bilinear operator $H_{W}^{\kappa, \sigma}$ defined by

$$
\int H_{W}^{\kappa, \sigma}\left(f_{1}, f_{2}\right)(x) f_{3}(x) d x=T_{W}^{\kappa}\left(f_{\sigma^{-1}(1)}, f_{\sigma^{-1}(2)}, f_{\sigma^{-1}(3)}\right) .
$$


We will prove Theorem 1 in two steps: The first step is to prove the following proposition:

Proposition 1. Let $1<r_{1}, r_{2}<2$ and assume

$$
\frac{1}{r}:=\frac{1}{r_{1}}+\frac{1}{r_{2}}>\frac{3}{2} \text {. }
$$

Then there is a constant $C$ such that for all $\kappa, \sigma$ as above and all $f_{1}, f_{2} \in C^{\Delta}(\mathbb{R})$

$$
\left\|H_{W}^{\kappa, \sigma}\left(f_{1}, f_{2}\right)\right\|_{r} \leq C\left\|f_{1}\right\|_{r_{1}}\left\|f_{2}\right\|_{r_{2}}
$$

The second step consists of an interpolation argument which is given in the appendix.

\section{Proof of Proposition 1.}

By Marcinkiewicz interpolation (see [3]) it suffices to prove the corresponding weak type estimate instead of (10). By homogeneity here we use that $\kappa$ was arbitrary - and linearity it suffices to prove that for $\left\|f_{1}\right\|_{r_{1}}=\left\|f_{2}\right\|_{r_{2}}=1$ we have

$$
\left|\left\{x: H_{W}^{\kappa, \sigma}\left(f_{1}, f_{2}\right)(x)>1\right\}\right| \leq C .
$$

Fix such $f_{1}$ and $f_{2}$ and define

$$
E:=\left\{x: \max \left\{M_{r_{1}}^{\Delta} f_{1}(x), M_{r_{2}}^{\Delta} f_{2}(x)\right\} \geq 1\right\} .
$$

Here we have set

$$
M_{p}^{\Delta} f(x):=\left(\sup _{I: \text { dyadic }, x \in I} \frac{1}{|I|} \int_{I}|f(x)|^{p} d x\right)^{1 / p} .
$$

By the maximal theorem the measure of $E$ is bounded by a universal constant, hence it suffices to prove a weak type estimate outside the set $E$; i.e., since each $v_{\imath, P}$ is supported on $I_{P}$, it suffices to prove a universal bound on the measure of the set

$$
F:=\left\{x: \sum_{P \in \mathbf{P}: I_{P} \not \subset E} \frac{1}{\sqrt{\left|I_{P}\right|}}\left\langle v_{\sigma(1), P}, f_{1}\right\rangle\left\langle v_{\sigma(2), P}, f_{2}\right\rangle v_{\sigma(3), P}(x)>1\right\} .
$$


For this we can assume that the measure of $F$ is larger than 1 . Let $f_{3}$ be the characteristic function of $F$, divided by $|F|^{1 / 2}$. It is easy to see that $f_{3} \in C^{\Delta}(\mathbb{R})$ and we have

$$
|F|^{1 / 2} \leq \sum_{P \in \mathbf{P}: I_{P} \not \subset E} \frac{1}{\sqrt{\left|I_{P}\right|}}\left\langle v_{\sigma(1), P}, f_{1}\right\rangle\left\langle v_{\sigma(2), P}, f_{2}\right\rangle\left\langle v_{\sigma(3), P}, f_{3}\right\rangle .
$$

Now the following lemma, applied with $r_{3}:=2$ and $f_{1}, f_{2}, f_{3}, r_{1}, r_{2}$ as above, implies that $|F|$ is bounded. Observe that for these data $E_{3}=\varnothing$, hence the set $E$ in the lemma coincides with the set $E$ above.

Lemma 4. Let $1<r_{1}, r_{2}, r_{3} \leq 2$ with

$$
1<\frac{1}{r_{1}}+\frac{1}{r_{2}}+\frac{1}{r_{3}}<2
$$

Then there is a constant $C$ such that the following holds: Let $f_{1}, f_{2}, f_{3} \in$ $C^{\Delta}(\mathbb{R})$ with

$$
\left\|f_{1}\right\|_{r_{1}}=\left\|f_{2}\right\|_{r_{2}}=\left\|f_{3}\right\|_{r_{3}}=1
$$

Define

$$
E_{\imath}:=\left\{x: M_{r_{\imath}}^{\Delta} f_{\imath}(x) \geq 1\right\}
$$

and $E:=E_{1} \cup E_{2} \cup E_{3}$. Then

$$
\sum_{P \in \mathbf{P}: I_{P} \not \subset E} \frac{1}{\sqrt{\left|I_{P}\right|}}\left|\left\langle v_{\sigma(1)}, f_{1}\right\rangle\left\langle v_{\sigma(2)}, f_{2}\right\rangle\left\langle v_{\sigma(3)}, f_{3}\right\rangle\right| \leq C .
$$

It remains to prove this lemma. By symmetry we can assume that $\sigma$ is the identity. First observe that under the hypotheses of the lemma it suffices to prove that for any finite subset $\mathbf{Q} \subset\left\{P \in \mathbf{P}: I_{P} \not \subset E\right\}$, such that $\left\langle v_{1, P}, f_{1}\right\rangle\left\langle v_{2, P}, f_{2}\right\rangle\left\langle v_{3, P}, f_{3}\right\rangle \neq 0$ for all $P \in \mathbf{Q}$, we have

$$
\sum_{P \in \mathbf{Q}}\left|I_{P}\right|^{-1 / 2}\left|\left\langle v_{1, P}, f_{1}\right\rangle\left\langle v_{2, P}, f_{2}\right\rangle\left\langle v_{3, P}, f_{3}\right\rangle\right| \leq C
$$

This inequality is the conclusion of Lemma 1 applied to the set $\mathbf{Q}$ and the functions $a_{\jmath}$ defined by

$$
a_{\jmath}(P)=\left|\left\langle v_{\jmath}, P, f_{\jmath}\right\rangle\right| .
$$


It remains to verify the two hypotheses of Lemma 1 with $s_{\jmath}:=r_{\jmath}{ }^{\prime}+\varepsilon$ for some small $\varepsilon$, and $B$ some number which will evolve from the estimates below.

\section{Verification of Lemma 1.1).}

Let $\imath \neq \jmath$. Fix a tree $T$ as in Hypothesis 1. It suffices to prove

$$
\left\|\left(\sum_{P \in T} \frac{\left|\left\langle f, v_{\jmath, P}\right\rangle\right|^{2}}{\left|I_{P}\right|} \mathbf{1}_{I_{P}}\right)^{1 / 2}\right\|_{t} \leq C\|f\|_{t}
$$

for all $1<t \leq 2$. Namely, if this is true, we apply it to $f=f_{\jmath} \mathbf{1}_{I_{T}}$ and obtain with Hölder's inequality

$$
\left\|\left(\sum_{P \in T} \frac{\left|\left\langle f, v_{\jmath, P}\right\rangle\right|^{2}}{\left|I_{P}\right|} \mathbf{1}_{I_{P}}\right)^{1 / 2}\right\|_{1} \leq C\left|I_{T}\right| \inf _{z \in I_{T}} M_{t}^{\Delta} f(z) .
$$

If we set $t:=r_{\jmath}$, then the right hand side is bounded by $C\left|I_{T}\right|$, since $I_{T} \not \subset E$. Hence Hypothesis 1 is satisfied.

By standard square function techniques it suffices to prove the estimate

$$
\left\|\sum_{P \in T} \varepsilon(P)\left\langle f, v_{\jmath, P}\right\rangle w_{p_{\jmath}(P)}\right\|_{t} \leq C\|f\|_{t}
$$

uniformly for all functions $\varepsilon: T \longrightarrow\{-1,1\}$ and all functions $f \in$ $C^{\Delta}(\mathbb{R})$.

First we assume that $\jmath \neq 3$ and prove this estimate by real interpolation. For $t=2$ it follows simply from the fact that the rectangles $\left\{p_{\jmath}(P): P \in T\right\}$ are pairwise disjoint. It remains to prove the weak type estimate

$$
\left|\left\{x: \sum_{P \in T} \varepsilon(P)\left\langle f, w_{p_{\jmath}(P)}\right\rangle w_{p_{\jmath}(P)}(x) \geq \lambda\right\}\right| \leq C\|f\|_{1} \lambda^{-1} .
$$

We fix $\lambda>0$ and split $f$ into a good function $g$ and a bad function $b$ as follows: Let $E$ be the set where the maximal function $M_{1}^{\Delta} f$ is larger than $\lambda$. Let $\left\{I_{n}\right\}_{n=1}^{N}$ be the set of maximal dyadic intervals contained in $E$. Define

$$
b_{n}:=\mathbf{1}_{I_{n}}\left(f-\lambda_{n} w_{p_{T}}\right),
$$


where $p_{T}$ is the top of the tree $T$ and $\lambda_{n}$ is chosen so that $b_{n}$ is orthogonal to $w_{p_{T}}$. Define $b=\sum_{n=1}^{N} b_{n}$ and $g=f-b$. It suffices to prove estimate (13) for $g$ and $b$ separately. Since $g$ is obviously bounded by $C \min \left\{\lambda, M_{1}^{\Delta} f(x)\right\}$, the estimate for $g$ follows from the previously proved $L^{2}$ estimate.

On the other hand,

$$
\sum_{P \in T} \varepsilon(P)\left\langle b_{n}, w_{p_{\jmath}(P)}\right\rangle w_{p_{\jmath}(P)}
$$

is supported on $4 I_{n}$. This is because if $I_{p_{\jmath}(P)}$ is larger than $4 I_{n}$, then there is a tile $q$ with $I_{q}=I_{n}, q<p_{\jmath}(P)$, and $q<p_{\jmath}\left(P_{T}\right)$. Hence $w_{p_{\jmath}(P)}$ and $w_{p_{T}}$ are multiples of each other on the interval $I_{q}$, and therefore $w_{p_{J}(P)}$ and $b_{n}$ are orthogonal.

This proves the weak type estimate for the bad function and thus finishes the proof of Hypothesis 1 in the case $\jmath \neq 3$.

Now assume $\jmath=3$. Instead of (12) we prove the dual estimate

$$
\left\|\sum_{P \in T} \varepsilon(P)\left\langle f, w_{p_{\jmath}(P)}\right\rangle v_{\jmath, P}\right\|_{t^{\prime}} \leq C\|f\|_{t^{\prime}} .
$$

If we replace $f$ by

$$
\sum_{P \in T} \varepsilon(P)\left\langle f, w_{p_{\jmath}(P)}\right\rangle w_{p_{\jmath}(P)}
$$

which by the ideas used in the case $\jmath \neq 3$ satisfies

$$
\left\|\sum_{P \in T} \varepsilon(P)\left\langle f, w_{p_{\jmath}(P)}\right\rangle w_{p_{\jmath}(P)}\right\|_{t^{\prime}} \leq C\|f\|_{t^{\prime}},
$$

we see that it suffices to prove

$$
\left\|\sum_{P \in T}\left\langle f, w_{p_{\jmath}(P)}\right\rangle v_{\jmath, P}\right\|_{t^{\prime}} \leq C\|f\|_{t^{\prime}} .
$$

This in turn follows by the maximal theorem from the pointwise estimate

$$
\left|\sum_{P \in T}\left\langle f, w_{p_{\jmath}(P)}\right\rangle v_{\jmath, P}(x)\right| \leq C M_{2}^{\Delta} f(x) .
$$

To prove this pointwise estimate, it suffices to prove

$$
\left|f_{k}\right| \leq C M_{2}^{\Delta} f,
$$


for all $k \in \mathbb{Z}$, where

$$
f_{k}:=\sum_{P \in T:\left|I_{P}\right| \geq 2^{k}}\left\langle f, w_{p_{\jmath}(P)}\right\rangle w_{p_{\jmath}(P)} .
$$

Fix $x \in \mathbb{R}$ and $k \in \mathbb{Z}$. Let $I_{k}$ and $I_{k-2}$ be the dyadic intervals containing $x$ of length $2^{k}$ and $2^{k-2}$ respectively. Then the functions $w_{p_{\jmath}(P)}$ with $I \subset I_{P},\left|I_{P}\right| \geq 2^{k}$ are multiples of each other on the interval $I_{k-2}$. Hence $f_{k}$ is of constant modulus on $I_{k-2}$, and we have

$$
\left|I_{k}\right|^{1 / 2}\left|f_{k}(x)\right| \leq C\left\|f_{k}\right\|_{L^{2}\left(I_{k}\right)} \text {. }
$$

It is easy to see that $f_{k}$ is orthogonal to $f-f_{k}$ on the interval $I_{k}$. Hence the right hand side of (15) can be estimated by $\|f\|_{L^{2}(I)}$. This proves (14) and completes the verification of Lemma 1.1).

\section{Verification of Lemma 1.2).}

Let $\mathbf{P}^{\prime} \subset \mathbf{Q}$ be a set of quartiles as in Hypothesis 2, i.e., $\mathbf{P}^{\prime}$ is a disjoint union of trees of type $\imath$

$$
\mathbf{P}^{\prime}:=\bigcup_{T \in \mathcal{F}} T
$$

such that the set $\left\{p_{\jmath}(P): P \in \mathbf{P}^{\prime}\right\}$ is a set of pairwise disjoint rectangles and, for each $T \in \mathcal{F}$, we have

$$
\left\|\left(\sum_{P \in T} \frac{\left|\left\langle f_{\jmath}, v_{\jmath}, P\right\rangle\right|^{2}}{\left|I_{P}\right|} \mathbf{1}_{I_{P}}\right)^{1 / 2}\right\|_{1} \geq 2^{m / s_{\jmath}}\left|I_{T}\right| .
$$

Here $\jmath \neq \imath$ and $m \in \mathbb{Z}$. Define the counting function

$$
N:=\sum_{T \in \mathcal{F}} \mathbf{1}_{I_{T}}
$$

We have to estimate the $L_{1}$-norm of the counting function $N$. Fix $\lambda \geq 0$ and consider the set

$$
E_{\lambda}:=\left\{x: M_{r_{\jmath}-\delta}^{\Delta} f_{\jmath}(x) \leq \gamma \lambda^{1 / r_{\jmath}} \text { and } N(x) \geq \lambda\right\}
$$


for some small constants $\gamma=\gamma\left(m, s_{\jmath}\right)>0$ and $\delta>0$ to be specified later. The set $E_{\lambda}$ is clearly contained in the set

$$
F_{\lambda}:=\left\{N(x) \geq \frac{\lambda}{4}\right\} .
$$

Let $I$ be a maximal dyadic interval contained in $F_{\lambda}$ and assume $I \cap E_{\lambda} \neq$ $\varnothing$. Define

$$
N_{I}=\sum_{T \in \mathcal{F}: I_{T} \subset I} \mathbf{1}_{I_{T}}
$$

Then $N-N_{I}$ is constant on $I$ and bounded by $\lambda / 4$, since otherwise the double of $I$ was also contained in $F_{\lambda}$, a contradiction to the maximality of $I$.

We assume the following inequality, which we will prove later

$$
\left|\left\{N_{I}(x) \geq \frac{\lambda}{4}\right\}\right| \leq C|I|\left(2^{-m / s_{\jmath}} \lambda^{-1 / s_{\jmath}} \inf _{x \in I} M_{r_{\jmath}-\delta}^{\Delta} f(x)\right)^{r_{\jmath}-\delta} .
$$

Since $I \cap E_{\lambda} \neq \varnothing$, the infimum on the right hand side is bounded by $\gamma \lambda^{1 / r_{3}}$. Moreover it is bounded by 1 , since otherwise $I$ was contained in the set where the maximal function $M_{r_{l}}^{\Delta} f$ is larger than 1, and hence $N_{I}=0$, which is impossible because $I \cap E_{\lambda} \neq \varnothing$.

Maximizing the expression on the right hand side of the previous inequality over $\lambda$ gives

$$
\left|\left\{N_{I}(x) \geq \frac{\lambda}{4}\right\}\right| \leq C|I|\left(2^{-m / s_{\jmath}} \gamma^{r_{\jmath} / s_{\jmath}}\right)^{r_{\jmath}-\delta} .
$$

Now we pick $\gamma$ smaller than $C 2^{m / r_{\jmath}}$ for an appropriate small constant $C$, then we have

$$
\left|\left\{N_{I}(x) \geq \frac{\lambda}{4}\right\}\right| \leq \frac{|I|}{100}
$$

Taking unions we obtain

$$
\left|E_{\lambda}\right| \leq \frac{\left|F_{\lambda}\right|}{100}
$$

Now we have

$$
\begin{aligned}
\|N\|_{1} & =\int_{\lambda=0}^{\infty}|\{N(x) \geq \lambda\}| d \lambda \\
& \leq \int\left|\left\{M_{r_{\jmath}-\delta}^{\Delta} f_{\jmath}(x) \geq \gamma \lambda^{1 / r_{\jmath}}\right\}\right| d \lambda+\int\left|E_{\lambda}\right| d \lambda \\
& \leq \gamma^{-r_{\jmath}}\left\|M_{r_{\jmath}-\delta}^{\Delta} f_{\jmath}\right\|_{r_{\jmath}}^{r_{\jmath}}+\frac{1}{100} \int\left|\left\{N(x) \geq \frac{\lambda}{4}\right\}\right| d \lambda \\
& \leq C 2^{-m}\left\|f_{\jmath}\right\|_{r_{\jmath}}+\frac{1}{10}\|N\|_{1} .
\end{aligned}
$$


This gives the appropriate bound on the counting function. Therefore it remains to prove (17).

Pick a $\mu \geq 1$ and define

$$
E_{\mu}:=\left\{x: N_{I}(x) \geq \mu\right\} .
$$

Define

$$
\begin{gathered}
\mathcal{F}_{I, \mu}:=\left\{T \in \mathcal{F}: I_{T} \subset I, I_{T} \not \subset E_{\mu}\right\}, \\
\mathbf{P}_{I, \mu}:=\bigcup_{T \in \mathcal{F}_{I, \mu}} T, \quad N_{I, \mu}:=\sum_{T \in T_{I, \mu}} \mathbf{1}_{I_{T}} .
\end{gathered}
$$

It is easy to see from the dyadic property of all intervals $I_{P}$ that $\left\|N_{I, \mu}\right\|_{\infty} \leq \mu$.

We introduce some measure spaces: The first one is the set $\mathbf{P}_{I, \mu}$ endowed with counting measure. The second one, $\mathcal{I}$, is as a set the abstract disjoint union of the sets $I_{T}, T \in \mathcal{F}_{I, \mu}$, where each of the $I_{T}$ is endowed with Lebesgue measure normalized such that $I_{T}$ has measure 1. The third one is $\mathcal{F}_{I, \mu}$ with counting measure. The fourth space is simply $\mathbb{R}$ with Lebesgue measure.

Now we consider functions on the cartesian product of these measure spaces,

$$
f: \mathbb{R} \times \mathcal{F}_{I, \mu} \times \mathcal{I} \times \mathbf{P}_{I, \mu} \longrightarrow \mathbb{R}
$$

and define norms on these functions by

$$
\|f\|_{p, q, r, s}:=\|\|\|\| f\left\|_{L^{s}\left(\mathbf{P}_{I, \mu}\right)}\right\|_{L^{r}(\mathcal{I})}\left\|_{L^{q}\left(\mathcal{F}_{I, \mu}\right)}\right\|_{L^{p}(\mathbb{R})} .
$$

Define the linear operator $S$ mapping functions on $\mathbb{R}$ to functions on $\mathbb{R} \times \mathcal{F}_{I, \mu} \times \mathcal{I} \times \mathbf{P}_{I, \mu}$ by

$S f(y, T, x, P)= \begin{cases}\frac{\left\langle f, w_{p_{\jmath}(P)}\right\rangle}{\left|I_{P}\right|^{1 / 2}}, & \text { if } y \in I_{T}, P \in T, \text { and } x \in I_{P} \subset I_{T}, \\ 0, & \text { otherwise. }\end{cases}$

Here the condition $x \in I_{P} \subset I_{T}$ means that $x$ is contained in the piece $I_{T}$ of $\mathcal{I}$ and in addition $x \in I_{P}$, where $I_{P}$ is naturally identified with a subset of this piece $I_{T}$. We have

$$
\begin{aligned}
\|S f\|_{2,2,2,2} & =\left(\int_{\mathbb{R}} \sum_{T \in \mathcal{F}_{I, \mu}} \mathbf{1}_{I_{T}}(y) \frac{1}{\left|I_{T}\right|} \int_{I_{T}} \sum_{P \in T} \frac{\left|\left\langle f, v_{\jmath, P}\right\rangle\right|^{2}}{\left|I_{P}\right|} \mathbf{1}_{I_{P}}(x) d x d y\right)^{1 / 2} \\
& =\left(\sum_{P \in \mathbf{P}_{I, \mu}}\left|\left\langle f, v_{\jmath, P}\right\rangle\right|^{2}\right)^{1 / 2} \\
& \leq C \log (1+\mu)^{2}\|f\|_{2} .
\end{aligned}
$$


If $\jmath \neq 3$, then the last inequality follows simply from the orthogonality of the $v_{\jmath, P}=w_{p_{\jmath}(P)}$. We postpone the proof of inequality (18) in the case $\jmath=3$ to the next section.

Moreover we have for small $\delta>0$

$$
\begin{aligned}
& \|S f\|_{1+2 \delta, \infty, 1,2} \\
& =\left(\int _ { \mathbb { R } } \operatorname { s u p } _ { T \in \mathcal { F } _ { I , \mu } : y \in I _ { T } } \left(\frac { 1 } { | I _ { T } | } \int _ { I _ { T } } \left(\sum_{P \in T} \frac{\left|\left\langle f, v_{\jmath, P}\right\rangle\right|^{2}}{\left|I_{P}\right|}\right.\right.\right. \\
& \left.\left.\left.\cdot \mathbf{1}_{I_{P}}(x)\right)^{1 / 2} d x\right)^{1+2 \delta} d y\right)^{1 /(1+2 \delta)} .
\end{aligned}
$$

Using (11) with $t=1+\delta$ we can bound this by

$$
\begin{aligned}
& \leq C\left(\int_{\mathbb{R}} \sup _{T \in \mathcal{F}_{I, \mu}: y \in I_{T}}\left(\inf _{z \in I_{T}} M_{1+\delta}^{\Delta} f(z)\right)^{1+2 \delta} d y\right)^{1 /(1+2 \delta)} \\
& \leq C\left(\int_{\mathbb{R}}\left(M_{1+\delta}^{\Delta} f(y)\right)^{1+2 \delta} d y\right)^{1 /(1+2 \delta)} \\
& \leq C\|f\|_{1+2 \delta} .
\end{aligned}
$$

The last line followed from the maximal theorem.

Interpolation and Hölder's inequality in the third exponent gives for a different small $\delta$

$$
\|S f\|_{r_{\jmath}-\delta, s_{\jmath}-\delta, 1,2} \leq C \log (1+\mu)^{2}\|f\|_{r_{\jmath}-\delta} .
$$

We replace in this inequality $f$ by $f \mathbf{1}_{I}$, which does not change the left hand side of this inequality. With the assumption (16) this gives

$$
\begin{aligned}
\left(\int_{\mathbb{R}}\left(\sum_{T \in \mathcal{F}}\left(\mathbf{1}_{I_{T}}(y) 2^{m / s_{\jmath}}\right)^{s_{\jmath}-\delta}\right)^{\left(r_{\jmath}-\delta\right) /\left(s_{\jmath}-\delta\right)} d x\right)^{1 /\left(r_{\jmath}-\delta\right)} \\
=\left\|N_{I, \mu}^{1 /\left(s_{\jmath}-\delta\right)}\right\|_{r_{\jmath}-\delta} 2^{m / s_{\jmath}} \\
\leq C \log (1+\mu)^{2}\left\|f \mathbf{1}_{I}\right\|_{r_{\jmath}-\delta} .
\end{aligned}
$$

This gives the weak type estimate

$$
\begin{aligned}
\left|\left\{x: N_{I} \geq \mu\right\}\right| & =\left|\left\{x: N_{I, \mu} \geq \mu\right\}\right| \\
& \leq C\left(\mu^{-1 /\left(s_{\jmath}-\delta\right)} \log (1+\mu)^{2} 2^{-m / s_{\jmath}}\left\|f \mathbf{1}_{I}\right\|_{r_{\jmath}-\delta}\right)^{r_{\jmath}-\delta} \\
& \leq C\left(\mu^{-1 / s_{\jmath}} 2^{-m / s_{\jmath}}\left\|f \mathbf{1}_{I}\right\|_{r_{\jmath}-\delta}\right)^{r_{\jmath}-\delta} .
\end{aligned}
$$


Since $\mu \geq 1$ was arbitrary and $N_{I}$ takes only integer values, this proves (17) and finishes the verification of Lemma 1.2).

It remains to prove inequality (18) in the case $\jmath=3$.

Proof of inequality (18) in The CASE $\jmath=3$. It suffices to prove for all functions $f \in C^{\Delta}(\mathbb{R})$

$$
\left\|\sum_{P \in \mathbf{P}_{I, \mu}}\left\langle f, w_{p_{\jmath}(P)}\right\rangle v_{\jmath}, P\right\|_{2} \leq C \log (1+\mu)^{2}\|f\|_{2} .
$$

Namely, this implies by duality

$$
\left\|\sum_{P \in \mathbf{P}_{I, \mu}}\left\langle f, v_{\jmath}, P\right\rangle w_{p_{\jmath}(P)}\right\|_{2} \leq C \log (1+\mu)^{2}\|f\|_{2} .
$$

which implies (18) by orthogonality of the $w_{p_{J}(P)}$.

We prove (20). Let $\mathcal{I}$ be the set of intervals $I_{T}$ with $T \in \mathcal{F}_{I, \mu}$. Let $\mathcal{I}_{1}$ be the set of maximal intervals in $\mathcal{I}$ with respect to set inclusion, and define $\mathcal{I}_{\nu}$ for $\nu=2,3, \ldots$ to be the set of maximal intervals in

$$
\mathcal{I} \backslash \bigcup_{\nu^{\prime}<\nu} I_{\nu^{\prime}}
$$

From the dyadic property of the intervals $I_{T}$ with $T \in \mathcal{F}_{I, \mu}$ we conclude that for every $J \in \mathcal{I}_{\nu}, \nu>1$, there is a $J^{\prime} \in \mathcal{I}_{\nu-1}$ with $J \subset J^{\prime}$. Since the counting function $N_{I, \mu}$ is bounded by $\mu$, we conclude that $I_{\nu}$ is empty for $\mu>\nu$.

Let $\mathbf{P}_{\nu}$ be the set of all tiles $p \in \mathbf{P}_{I, \mu}$ with $I_{p} \subset J$ for some $J \in \mathcal{I}_{\nu}$, but $I_{p} \not \subset J^{\prime}$ for all $J^{\prime} \in \mathcal{I}_{\nu+1}$. Define $\nu(x)$ so that the left hand side of (20) is bounded by

$$
\begin{aligned}
\left.\| \sup _{\nu} \mid \sum_{n=1}^{\nu}\left(\sum_{P \in \mathbf{P}_{n}}\left\langle f, w_{p_{\jmath}(P)}\right\rangle w_{p_{\jmath}(P)}\right)\right) \mid \|_{2} \\
+\left\|\sum_{P \in \mathbf{P}_{\nu(x)}:\left|I_{p}\right| \geq 2^{\kappa(x)}}\left\langle f, w_{p_{\jmath}(P)}\right\rangle w_{p_{\jmath}(P)}\right\|_{2} .
\end{aligned}
$$

By Rademacher-Menshov, the first term in this sum is bounded by $C \log (\mu+1)\|f\|_{2}$, which is the desired estimate for this summand. The second summand can be estimated by

$$
\left(\sum_{\nu}\left\|\sup _{k}\left|\sum_{P \in \mathbf{P}_{\nu}:\left|I_{p}\right| \geq 2^{k}}\left\langle f_{\nu}, w_{p_{\jmath}(P)}\right\rangle w_{p_{\jmath}(P)}\right|\right\|_{2}^{2}\right)^{1 / 2},
$$


where

$$
f_{\nu}:=\sum_{P \in \mathbf{P}_{\nu}}\left\langle f, w_{p_{\jmath}(P)}\right\rangle w_{p_{\jmath}(P)} .
$$

Since the functions $f_{\nu}$ are orthogonal as $\nu$ varies, it suffices to prove for a fixed $\nu$

$$
\left\|\sup _{k}\left|\sum_{P \in \mathbf{P}_{\nu}:\left|I_{p}\right|>2^{k}}\left\langle f, w_{p_{\jmath}(P)}\right\rangle w_{p_{\jmath}(P)}\right|\right\|_{2} \leq C \log (\mu+1)^{2}\|f\|_{2} .
$$

We split the set $\mathbf{P}_{\nu}$ further. Let $J$ be an interval in $\mathcal{I}_{\nu}$. By a trivial splitting of $\mathbf{P}_{\nu}$ we can assume that all $P \in \mathbf{P}_{\nu}$ satisfy $I_{P} \subset J$. Then, if $P \in \mathbf{P}_{\nu}$, we necessarily have $P \in T$ for some tree $T \in \mathcal{F}_{I, \mu}$ with $J \subset I_{T}$. Hence we can find a collection of at most $\mu$ trees $T \in \mathcal{F}_{I, \mu}$ such that $P_{\nu}$ is contained in the union of these trees. For each tree $T$ in this collection pick a top frequency $\xi \in \omega_{T}$, and let $\Xi$ be the set of these frequencies.

For each integer $k$ with $2^{k}<|J|$ consider the collection $\Omega_{k}$ of all dyadic intervals of length $2^{-k}$ which have nonempty intersection with $\Xi$. Call $k$ an exceptional value if if the cardinality of $\Omega_{k+4}$ is larger than the cardinality of $\Omega_{k-4}$. There are at most $8 \mu$ exceptional values. Pick a chain of integers $k_{0}<k_{1}<k_{2}<\cdots<k_{8 \mu}$ such that all exceptional values appear in this chain.

We can estimate the left hand side of (21) by

$$
\begin{aligned}
\| \sup _{m} \mid & \sum_{p \in \mathbf{P}_{\nu}:\left|I_{P}\right|>2^{k_{m}}}\left\langle f, w_{p_{\jmath}(P)}\right\rangle w_{p_{\jmath}(P)} \mid \|_{2} \\
& +\left\|\sup _{m} \sup _{k_{m-1}<k \leq k_{m}} \mid \sum_{p \in \mathbf{P}_{\nu}: 2^{k_{m}} \geq\left|I_{P}\right|>2^{k}}\left\langle f, w_{p_{\jmath}(P)}\right\rangle w_{p_{\jmath}(P)}\right\|_{2} .
\end{aligned}
$$

Again by Rademacher-Menshov the first summand is bounded by $C \log (\mu+1)\|f\|_{2}$.

To estimate the second summand it suffices by a similar argument as before to prove for each $m$

$$
\begin{array}{r}
\left\|\sup _{k_{m}-1<k \leq k_{m}}\left|\sum_{p \in \mathbf{P}_{\nu}: 2^{k_{m}} \geq\left|I_{P}\right|>2^{k}}\left\langle f, w_{p_{\jmath}(P)}\right\rangle w_{p_{\jmath}(P)}\right|\right\|_{2} \\
\leq C \log (\mu+1)^{2}\|f\|_{2} .
\end{array}
$$

If $k_{m-1}=k_{m}-1$, then this estimate is trivial. Therefore assume that $k_{m-1}<k_{m}-1$. 
We claim that for $k_{m-1}<k \leq k_{m}$ we have

$$
\begin{aligned}
\sum_{p \in \mathbf{P}_{\nu}: 2^{k_{m}} \geq\left|I_{P}\right|>2^{k}}\left\langle f, w_{p_{\jmath}(P)}\right\rangle w_{p_{\jmath}(P)} & \\
& =\Pi_{k+k_{0}} \sum_{p \in \mathbf{P}_{\nu}: 2^{k_{m}} \geq\left|I_{P}\right|>2^{k_{m}-1}}\left\langle f, w_{p_{\jmath}(P)}\right\rangle w_{p_{\jmath}(P)} .
\end{aligned}
$$

Here $\Pi_{k+k_{0}}$ denotes the projection onto the subspace of $L^{2}(\mathbb{R})$ corresponding to all points in the Walsh phase plane whose frequency coordinate is contained in the union of intervals in $\Omega_{k-k_{0}}$, where $k_{0} \in\{0,1\}$ depends only on $\jmath$ and $\imath$. For the definition and properties of subspaces associated to sets in the Walsh phase plane (see [11]).

We prove the claim in the case $\imath=1$ and $\jmath=2$, the other cases being similar. In this case we have $k_{0}=0$. Let $F_{k}$ be the union of all intervals in $\Omega_{k}$. To prove inequality (23) we have to show that for all $P \in \mathbf{P}_{\nu}$ with $I_{P}=2^{k}$ we have $\omega_{p_{\jmath}(P)} \subset F_{k-1}$ and $\omega_{p_{\jmath}(P)} \cap \subset F_{k}=\varnothing$. However it is clear that $\omega_{p_{\imath}(P)}$ contains a $\xi \in \Xi$, hence $\omega_{\imath(P)} \cup \omega_{\jmath(P)}$ is a dyadic interval of length $2^{-k+1}$ having nonempty intersection with $\Xi$ and therefore being contained in $F_{k-1}$. Moreover, $\omega_{p_{\jmath}(P)} \cap \subset F_{k}=$ $\varnothing$, because $k$ is not exceptional and therefore the two neighbouring intervals $\omega_{p_{\imath}(P)}$ and $\omega_{p_{\jmath}(P)}$ can not be both in $\Omega_{k}$.

Now the claim (23) shows that inequality (22) is a direct consequence of the following Lemma which is a version of a lemma by Bourgain (see [11]):

Lemma 5 (Bourgain). Let $\Xi \subset \mathbb{R}^{+}$. For each integer $k$ define $\Omega_{k}$ to be the set of dyadic intervals of length $2^{-k}$ which have nonempty intersection with $\Xi$. Define $\Pi_{k}$ to be the orthogonal projection onto the subspace of $L^{2}(\mathbb{R})$ associated to the set of all points in the phase plane whose frequency coordinate is contained in the union of the intervals in $\Omega_{k}$. Let $k<k^{\prime}$ be two integers such that $\Omega_{k}$ and $\Omega_{k^{\prime}}$ have the same cardinality. Define

$$
M_{\Xi} f(x):=\sup _{k<\kappa \leq k^{\prime}}\left|\Pi_{\kappa} f(x)\right|
$$

Then

$$
\left\|M_{\Xi} f\right\|_{2} \leq C \log (\sharp \Xi)^{2}\|f\|_{2} .
$$


Proof of Lemma 5. Following [1], we present a series of lemmata that leads to a proof of Lemma 5. The first lemma is a version of Doob's oscillation lemma for martingales and is obtained by methods of stopping times and square functions

Lemma 6 (Doob). Let $1<r<\infty$ and $f \in L^{r}(\mathbb{R})$. For each dyadic interval I let $m_{I} f$ denote the mean of $f$ on $I$. For $\lambda>0$ and $x \in \mathbb{R}$ let $M_{\lambda}(x)$ be the maximal number such that there is an increasing chain of dyadic intervals $x \in I_{1} \subset I_{2} \subset \cdots \subset I_{M_{\lambda}(x)}$ with

$$
\left|m_{I_{j}} f-m_{I_{j+1}} f\right| \geq \lambda \text {. }
$$

Then

$$
\left\|\lambda M_{\lambda}^{1 / 2}\right\|_{r} \leq C_{r}\|f\|_{r}
$$

For a proof of this Lemma we refer to [10]. With Lemma 6 we prove the following lemma due to Lépingle (see [9]):

Lemma 7 (Lépingle). Let $\varepsilon$ be small and $2-\varepsilon<p<s<2+\varepsilon, 2<s$. Let $f \in L^{p}(\mathbb{R})$. Then

$$
\begin{aligned}
& \int \sup \left\{\left(\sum_{j=1}^{J}\left|m_{I_{j}} f-m_{I_{j+1}} f\right|^{s}\right)^{1 / s}: J \in \mathbb{N}_{0},\right. \\
& \left.x \in I_{1} \subset I_{2} \subset \cdots \subset I_{J+1}\right\}^{p} d x \\
& \leq C(s-2)^{-1}\|f\|_{p}^{p} .
\end{aligned}
$$

Proof. By interpolation it suffices to prove this for $f$ being the characteristic function of a set $A$. Using the numbers $M_{\lambda}(x)$ defined in Lemma 6 we can estimate (24) by

$$
\int\left(\sum_{n=0}^{\infty} 2^{-n s} M_{2^{-n}}(x)\right)^{p / s} d x
$$

Now an easy calculation shows that this is bounded by

$$
\sum_{n=0}^{\infty} 2^{-n p} 2^{2 n p / s} \int 2^{-2 n p / s}\left(M_{2^{-n}}^{1 / 2}(x)\right)^{2 p / s} d x .
$$


By Lemma 6 this is bounded by

$$
\sum_{n=0}^{\infty} 2^{-n p(1-2 / s)}|A| \leq C\left(\frac{1}{2}-\frac{1}{s}\right)\|f\|_{p}^{p} .
$$

This proves Lemma 7 .

Next, we prove a vector valued version of Lemma 7 .

Lemma 8 (Lépingle, vector valued). Consider the Euclidean space $\mathbb{R}^{n}$ and let $f=\left(f_{1}, \ldots, f_{n}\right) \in L^{2}\left(\mathbb{R}, \mathbb{R}^{n}\right)$. Let $2<s$. For $\lambda>0, x \in \mathbb{R}$ let $M_{\lambda}(x)$ denote the minimal number of $\lambda$-balls necessary to cover the set $\left\{m_{I} f\right\}_{I \text { dyadic }: x \in I}$. Then

$$
\left\|\sup _{\lambda>0}\left(\lambda M_{\lambda}^{1 / s}\right)\right\|_{2}^{2} \leq C(s-2)^{-1} \sum_{\alpha=1}^{n}\left\|f_{\alpha}\right\|_{2}^{2} .
$$

For a proof of this lemma we calculate with $p=2$ in the previous lemma

$$
\begin{aligned}
& \left\|\sup _{\lambda>0}\left(\lambda M_{\lambda}^{1 / s}\right)\right\|_{2}^{2} \\
& \leq C \int \sup \left\{\left(\sum_{j=1}^{J}\left(\sum_{\alpha=1}^{n}\left|m_{I_{j}} f_{\alpha}-m_{I_{j+1}} f_{\alpha}\right|^{2}\right)^{s / 2}\right)^{1 / s}:\right. \\
& \left.\quad J \in \mathbb{N}_{0}, x \in I_{1} \subset I_{2} \subset \cdots \subset I_{J+1}\right\}^{2} d x \\
& \leq C \sum_{\alpha=1}^{n} \int \sup \left\{\left(\sum_{j=1}^{J}\left|m_{I_{j}} f_{\alpha}-m_{I_{j+1}} f_{\alpha}\right|^{s}\right)^{1 / s}:\right. \\
& \left.\quad J \in \mathbb{N}_{0}, x \in I_{1} \subset I_{2} \subset \cdots \subset I_{J+1}\right\}^{2} d x \\
& \leq C(s-2)^{-1} \sum_{\alpha=1}^{n}\left\|f_{\alpha}\right\|_{2}^{2} .
\end{aligned}
$$

This proves Lemma 8 .

Now we proceed to prove Lemma 5 . By passing to a subset of $\Xi$ if necessary we can assume that $\Omega_{k}$ and $\Omega_{k^{\prime}}$ have the same cardinality as $\Xi$. We enumerate $\Xi$ as $\xi_{1}, \ldots, \xi_{n}$. 
Let $x \in \mathbb{R}$. We use the following equality, which is an easy result of Walsh phase plane analysis as in [11]

$$
\Pi_{k} f(x)=\sum_{\alpha=1}^{n} m_{k}\left(f w_{\xi_{\alpha}}\right) w_{\xi_{\alpha}}
$$

where $w_{\xi}$ is the Walsh function of modulus 1 on $\mathbb{R}$ associated to the frequency $\xi$ and $m_{k}(f)(x)$ is the mean of $f$ over the dyadic interval of length $2^{k}$ which contains $x$.

Let $J$ be a dyadic interval of length $2^{k}$. For $s \in \mathbb{Z}$ pick a minimal collection $\mathbf{B}_{s, J}$ of $2^{s}$-balls covering the set

$$
\left\{\left(m_{\kappa} f_{1}(x), \ldots, m_{\kappa} f_{n}(x)\right): k<\kappa \leq k^{\prime}\right\},
$$

where $x \in J$ and the set clearly does not depend on the choice of $x$.

Define the function

$$
G:=\left(\sum_{\alpha=1}^{n}\left(M^{\Delta}\left(m_{k}\left(f w_{\xi_{\alpha}}\right)\right)\right)^{2}\right)^{1 / 2}
$$

where $M^{\Delta}$ denotes the dyadic Hardy Littlewood maximal function. The function $G$ is constant on dyadic inetrvals of length $J$, and we write $G_{J}$ for the value of $G$ on $J$.

If $s$ is larger that $2+\log _{2} G_{J}$, then the ball of radius $2^{s}$ centered at the origin covers the set $(25)$, and we pick $\mathbf{B}_{s, J}$ to just consist of this ball. For each ball $B \in \mathbf{B}_{s, J}$ pick a ball $B^{\prime} \in \mathbf{B}_{s+1, J}$ which has nonempty intersection with $B$. Let $d(B)=c(B)-c\left(B^{\prime}\right)$, where $c(B)$ denotes the center of $B$. Clearly the length of the vector $d(B)$ is less than $2^{s+2}$. We write $d_{\alpha}(B)$ for the $\alpha$-th coordinate of $d(B)$.

For each $k<\kappa \leq k^{\prime}$ we can find balls $B_{\kappa, s, J} \in \mathbf{B}_{s, J}$ such that for each $x \in J, 1 \leq \alpha \leq n$

$$
m_{\kappa}\left(f w_{\xi_{\alpha}}\right)(x):=\sum_{s \in \mathbb{Z}} d_{\alpha}\left(B_{\kappa, s, J}\right)
$$

Then we have

$$
\begin{aligned}
& \sup _{k<\kappa \leq k^{\prime}}\left|\sum_{\alpha=1}^{n} m_{k}\left(f w_{\xi_{\alpha}}\right) w_{\xi_{\alpha}}(x)\right| \\
& \leq \sup _{k<\kappa \leq k^{\prime}}\left|\sum_{\alpha=1}^{n} \sum_{s \in \mathbb{Z}} d_{\alpha}\left(B_{\kappa, s, J}\right) w_{\xi_{\alpha}}(x)\right|
\end{aligned}
$$




$$
\begin{aligned}
& \leq \sum_{s \in \mathbb{Z}} \max _{B \in \mathbf{B}_{s, J}}\left|\sum_{\alpha=1}^{n} d_{\alpha}(B) w_{\xi_{\alpha}}(x)\right| \\
& \leq C \sum_{s \in \mathbb{Z}} \min \left\{2^{s} n^{1 / 2},\left(\sum_{B \in \mathbf{B}_{s, J}}\left|\sum_{\alpha=1}^{n} d_{\alpha}(B) w_{\xi_{\alpha}}(x)\right|^{2}\right)^{1 / 2}\right\} .
\end{aligned}
$$

Hence we obtain

$$
\begin{aligned}
& \left\|\sup _{k<\kappa \leq k^{\prime}}\left|\sum_{\alpha=1}^{n} m_{\kappa}\left(f_{\alpha}\right) w_{\xi_{\alpha}}\right|\right\|_{L^{2}(J)} \\
& \quad \leq C \sum_{s \in \mathbb{Z}} \min \left\{2^{k / 2} 2^{s} n^{1 / 2},\left(\sum_{B \in \mathbf{B}_{s, J}}\left\|\sum_{\alpha=1}^{n} d_{\alpha}(B) w_{\xi_{\alpha}}\right\|_{L^{2}(J)}^{2}\right)^{1 / 2}\right\} .
\end{aligned}
$$

The functions $w_{\xi_{\alpha}}$ restricted to $J$ are pairwise orthogonal for $1 \leq \alpha \leq n$, hence we can estimate the previously displayed expression by

$$
\begin{aligned}
& \leq C 2^{k / 2} \sum_{\substack{s \in \mathbb{Z} \\
s \leq 2+\log _{2} G_{J}}} \min \left\{2^{s} n^{1 / 2}, 2^{s}\left|\mathbf{B}_{s, J}\right|^{1 / 2}\right\} \\
& \leq C\left\|\int_{0}^{4 G_{J}} \min \left\{n^{1 / 2}, M_{\lambda}^{1 / 2}\right\} d \lambda\right\|_{L^{2}(J)} .
\end{aligned}
$$

Hence

$$
\left\|\sup _{k<\kappa \leq k^{\prime}}\left|\sum_{\alpha=1}^{n} m_{\kappa}\left(f_{\alpha}\right) w_{\xi_{\alpha}}\right|\right\|_{L^{2}} \leq C\left\|\int_{0}^{4 G} \min \left\{n^{1 / 2}, M_{\lambda}^{1 / 2}\right\} d \lambda\right\|_{2} .
$$

Moreover,

$$
\begin{aligned}
& \int_{0}^{4 G(x)} \min \left\{n^{1 / 2}, M_{\lambda}^{1 / 2}\right\} d \lambda \\
& \leq G(x)+\int_{n^{-1 / 2} G(x)}^{4 G(x)} n^{1 / 2-1 / s} M_{\lambda}(x)^{1 / s} d \lambda \\
& \leq G(x)+C n^{1 / 2-1 / s} \log (1+n) \sup _{\lambda>0} \lambda M_{\lambda}(x)^{1 / s} .
\end{aligned}
$$

If we pick $s$ such that $1 / 2-1 / s$ is $\log (n+1)^{-1}$, then taking the $L^{2}$ norm in $x$ of the previously displayed expression and using Lemma 8 proves Lemma 5. 
This completes the proof of Proposition 1.

\section{Appendix: Interpolation.}

So far we have only proved some of the estimates which are claimed in Theorem 1. Now we prove the remaining estimates by interpolation.

Recall the definition of the trilinear form

$$
\begin{gathered}
T_{W}^{\kappa}: C^{\Delta}(\mathbb{R}) \times C^{\Delta}(\mathbb{R}) \times C^{\Delta}(\mathbb{R}) \longrightarrow \mathbb{R} \\
T_{W}^{\kappa}\left(f_{1}, f_{2}, f_{3}\right)=\sum_{P \in \mathbf{P}} \frac{1}{\sqrt{\left|I_{P}\right|}}\left\langle v_{1, P}, f_{1}\right\rangle\left\langle v_{2, P}, f_{2}\right\rangle\left\langle v_{3, P}, f_{3}\right\rangle .
\end{gathered}
$$

Also recall that for each permutation $\sigma$ of the set $\{1,2,3\}$ we have the bilinear operator $H_{W}^{\kappa, \sigma}$ defined by

$$
\int H_{W}^{\kappa, \sigma}\left(f_{1}, f_{2}\right)(x) f_{3}(x) d x=T_{W}^{\kappa}\left(f_{\sigma^{-1}(1)}, f_{\sigma^{-1}(2)}, f_{\sigma^{-1}(3)}\right) .
$$

Let $p_{1}, p_{2}, p_{3} \in \mathbb{R} \cup\{\infty\}$. We say that $T_{W}^{\kappa}$ is of type $\left(p_{1}, p_{2}, p_{3}\right)$ if there is a permutation $\sigma$ such that

$$
0<p_{\sigma(1)}, p_{\sigma(2)}, p_{\sigma(3)}{ }^{\prime} \leq \infty
$$

and there is a constant $C$ such that

$$
\left\|H_{W}^{\kappa, \sigma}(f, g)\right\|_{p_{\sigma(3)}^{\prime}} \leq C\|f\|_{p_{\sigma(1)}}\|g\|_{p_{\sigma(2)}},
$$

for all functions $f, g \in C^{\Delta}(\mathbb{R})$. Here $p^{\prime}$ denotes the conjugate exponent of $p$ defined by

$$
\frac{1}{p^{\prime}}+\frac{1}{p}=1
$$

We claim the following theorem, which implies Theorem 1.

Theorem 2. Let $\kappa \in C^{\Delta}(\mathbb{R})$. If

$$
\frac{1}{p_{1}}+\frac{1}{p_{2}}+\frac{1}{p_{3}}=1, \quad-\frac{1}{2}<\frac{1}{p_{1}}, \frac{1}{p_{2}}, \frac{1}{p_{3}}<1,
$$

then $T_{W}^{\kappa}$ is of type $\left(p_{1}, p_{2}, p_{3}\right)$. 
The following diagram shows the plane of all points $\left(1 / p_{1}, 1 / p_{2}\right.$, $\left.1 / p_{3}\right)$ with $1 / p_{1}+1 / p_{2}+1 / p_{3}=1$.

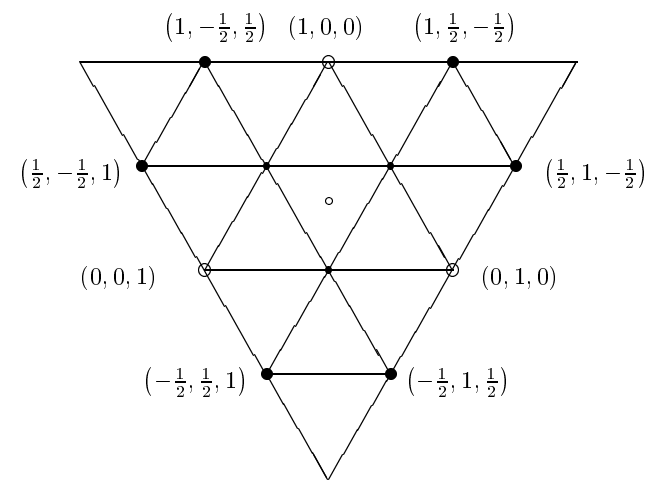

Let $A$ be the open interior of the convex hull of the six large filled circles in the above diagram. Theorem 2 states that $T_{W}^{\kappa}$ is of type $\left(p_{1}, p_{2}, p_{3}\right)$ for all $\left(1 / p_{1}, 1 / p_{2}, 1 / p_{3}\right) \in A$. The closed convex hull $B$ of the three large empty circles is the region in which condition (26) is satisfied for all permutations $\sigma$ and thus the type estimates (27) are equivalent for all six bilinear operators $H_{W}^{\kappa, \sigma}$. The remainder set $A \backslash B$ splits into three connected regions $D_{\jmath}$ such that the exponent $p_{\jmath}$ is negative in the region $D_{\jmath}$ for $\jmath=1,2,3$. In each of these regions, only two permutations $\sigma$ satisfy (26) and thus the estimate (27) makes sense only for the two corresponding bilinear operators $H_{W}^{\kappa, \sigma}$.

Proposition 1 proves the type estimates in each of the three triangles which are spanned by two adjacent large filled circles and the adjacent small filled circle in the above diagram.

Hence Theorem 2 follows from Proposition 1 and the following convexity lemma:

Lemma 9. Let $\left(1 / p_{1}, 1 / p_{2}, 1 / p_{3}\right)$ and $\left(1 / q_{2}, 1 / q_{2}, 1 / q_{3}\right)$ be two points in the region $A$ such that $p_{\imath}=q_{\imath}$ for some $\imath \in\{1,2,3\}$ and assume that $T_{W}^{\kappa}$ is of type $\left(p_{1}, p_{2}, p_{3}\right)$ and of type $\left(q_{1}, q_{2}, q_{3}\right)$. Then $T_{W}^{\kappa}$ is of type $\left(u_{1}, u_{2}, u_{3}\right)$ for all $\left(1 / u_{1}, 1 / u_{2}, 1 / u_{3}\right)$ on the line segment connecting $\left(1 / p_{1}, 1 / p_{2}, 1 / p_{3}\right)$ and $\left(1 / q_{2}, 1 / q_{2}, 1 / q_{3}\right)$.

We prove the lemma. The conclusion of the lemma follows immediately by complex interpolation as in [2], if there exists a $\sigma$ such that type $\left(p_{1}, p_{2}, p_{3}\right)$ and type $\left(q_{1}, q_{2}, q_{3}\right)$ can be expressed as estimates for $H_{W}^{\kappa, \sigma}$ (i.e., all $p_{\imath}, q_{\imath}$ are in $(1, \infty]$. This is the case if there is a 
$\jmath \in\{1,2,3\}$ such that both $\left(1 / p_{1}, 1 / p_{2}, 1 / p_{3}\right)$ and $\left(1 / q_{1}, 1 / q_{2}, 1 / q_{3}\right)$ are contained in the region $B \cup D_{\jmath}$.

Therefore we can assume that

$$
\left(\frac{1}{p_{1}}, \frac{1}{p_{2}}, \frac{1}{p_{3}}\right)
$$

and

$$
\left(\frac{1}{q_{1}}, \frac{1}{q_{2}}, \frac{1}{q_{3}}\right)
$$

are in different regions $D_{\jmath}$. Let $\sigma$ and $\tau$ be permutations such that

$$
\begin{gathered}
\left\|H_{W}^{\kappa, \sigma}(f, g)\right\|_{p_{\sigma(3)}^{\prime}} \leq C_{p}\|f\|_{p_{\sigma(1)}}\|g\|_{p_{\sigma(2)}}, \\
\left\|H_{W}^{\kappa, \tau}(f, g)\right\|_{q_{\tau(3)}^{\prime}} \leq C_{q}\|f\|_{q_{\tau(1)}}\|g\|_{q_{\tau(2)}},
\end{gathered}
$$

for all functions $f, g \in C^{\Delta}(\mathbb{R})$. By symmetry we can assume that $\sigma(1)=\tau(1)$, and then we necessarily have

$$
p_{\sigma(1)}=q_{\tau(1)} \cdot
$$

Let $C_{p}$ and $C_{q}$ be the optimal constants in the above estimates.

Pick two different points $\left(1 / u_{1}, 1 / u_{2}, 1 / u_{3}\right)$ and $\left(1 / v_{1}, 1 / v_{2}, 1 / v_{3}\right)$ on the line segment connecting the points $\left(1 / p_{1}, 1 / p_{2}, 1 / p_{3}\right)$ and $\left(1 / q_{1}\right.$, $\left.1 / q_{2}, 1 / q_{3}\right)$ such that $\left(1 / u_{1}, 1 / u_{2}, 1 / u_{3}\right)$ and $\left(1 / v_{1}, 1 / v_{2}, 1 / v_{3}\right)$ are both in the open interior of the region $B$ and the distance between $\left(1 / p_{1}\right.$, $\left.1 / p_{2}, 1 / p_{3}\right)$ and $\left(1 / u_{1}, 1 / u_{2}, 1 / u_{3}\right)$ is smaller than the distance between the points $\left(1 / p_{1}, 1 / p_{2}, 1 / p_{3}\right)$ and $\left(1 / v_{1}, 1 / v_{2}, 1 / v_{3}\right)$. It is easy to see that such points exist, because $\left(1 / p_{1}, 1 / p_{2}, 1 / p_{3}\right)$ and $\left(1 / q_{1}, 1 / q_{2}, 1 / q_{3}\right)$ are in different regions $D_{\jmath}$.

Let $f \in C^{\Delta}(\mathbb{R})$ be fixed. It is easy to see that there are constants $C_{u}$ and $C_{v}$, possibly depending on $f$, such that

$$
\begin{gathered}
\left\|H_{W}^{\kappa, \sigma}(f, g)\right\|_{u_{\sigma(3)}^{\prime}} \leq C_{u}\|f\|_{u_{\sigma(1)}}\|g\|_{u_{\sigma(2)}} \\
\left\|H_{W}^{\kappa, \sigma}(f, g)\right\|_{v_{\sigma(3)}^{\prime}} \leq C_{v}\|f\|_{v_{\sigma(1)}}\|g\|_{v_{\sigma(2)}}
\end{gathered}
$$

for all functions $g \in C^{\Delta}(\mathbb{R})$. Let $C_{u}$ and $C_{v}$ be the best constants in these inequalities. Assume to get a contradiction that $C_{v}$ is larger than $C_{p}$ and $C_{q}$. Then it follows by interpolation as in [2] between the 
estimates (28) and (31) that $C_{u}$ is smaller than $C_{v}$. However, we have by duality

$$
\begin{gathered}
\left\|H_{W}^{\kappa, \tau}(f, g)\right\|_{u_{\tau_{(3)}^{\prime}}^{\prime}} \leq C_{u}\|f\|_{u_{\tau(1)}}\|g\|_{u_{\tau(2)}} \\
\left\|H_{W}^{\kappa, \tau}(f, g)\right\|_{v_{\tau(3)}^{\prime}} \leq C_{v}\|f\|_{v_{\tau(1)}}\|g\|_{v_{\tau(2)}},
\end{gathered}
$$

for all $g \in C^{\Delta}(\mathbb{R})$, where the same constants $C_{u}$ and $C_{v}$ as above are optimal. Hence it follows by interpolation between the estimates (33) and (29) that $C_{v}$ is smaller than $C_{u}$ or $C_{q}$, a contradiction.

Hence $C_{v}$ is smaller than $C_{p}$ or $C_{q}$, which are independent of $f$. Hence $T_{W}^{\kappa}$ is of type $\left(v_{1}, v_{2}, v_{3}\right)$, and now the Lemma follows by interpolation between (28) and (31), and by interpolation between (29) and (33).

This completes the proof of Lemma 9, and therefore also the proof of theorems 2 and 1.

\section{References.}

[1] Bourgain, J., Pointwise ergodic theorems for arithmetic sets. Inst. Hautes Etudes Sci. Publ. Math. 69 (1989), 5-45.

[2] Calderon, A. P., Zygmund, A., A note on the interpolation of linear operators. Studia Math. 12 (1951), 194-204.

[3] Janson, S., On interpolation of multi-linear operators. Proceedings Lund 1986, Springer LNM 1302, 1988.

[4] Lacey, M., The bilinear maximal function maps into $L^{p}$ for $2 / 3<p \leq$ 1. Ann. Math. 151 (2000), 35-57.

[5] Lacey, M., Thiele, C., $L^{p}$ estimates on the bilinear Hilbert transform for $2<p<\infty$. Ann. Math. 146 (1997), 693-724.

[6] Lacey, M., Thiele, C., Bounds for the bilinear Hilbert transform on $L^{p}$. Proc. Nat. Acad. Sci. USA 94 (1997), 33-35.

[7] Lacey, M., Thiele, C., On Calderon's conjecture. Ann. Math. 149 (1999), 475-496.

[8] Lacey, M., Thiele, C., On the Calderon conjectures for the bilinear Hilbert transform. Proc. Nat. Acad. Sci. USA 95 (1998), 4828-4830.

[9] Lépingle, D., La variation d' ordre $p$ des semi-martingales. Z. Wahr. verw. Geb. 36 (1976), 295-316.

[10] Neveu, J., Martingales à temps discret. Masson, 1972.

[11] Thiele, C., Time-frequency analysis in the discrete phase plane. Ph. D. Thesis. Yale University, 1995. 
[12] Thiele, C., On the Bilinear Hilbert Transform. Habilitationsschrift, Universität Kiel, 1999.

Recibido: 20 de abril de 1.999

Christoph Thiele

Department of Mathematics University of California Los Angeles Los Angeles, CA 90095-1555, U.S.A.

thiele@math.ucla.edu 